\title{
Pseudocholinesterase Deficiency in Patient Undergoing Microlaringeal Surgery
}

\author{
Marinov Ts. ', M. Belitova', T. Popov², N. Nizamova², \\ J. Rangachev', D. Popova² \\ 'Department of Anesthesiology and Intensive Care; \\ University Hospital "Queen Giovanna” - ISUL; Medical University - Sofia \\ 2Department of ENT Surgery; University Hospital "Queen Giovanna” - ISUL; \\ Medical University - Sofia
}

\begin{abstract}
:
Introduction: Pseudocholinesterase deficiency is a genetic or acquired alteration in the metabolism of choline esters such as succinylcholine. Case description: A 70 years old female patient (body weight $48 \mathrm{~kg}$, height $157 \mathrm{~cm}$ ) was admitted to the hospital for microlaryngeal surgery. The preoperative interrogation revealed no significant personal or family history of adverse reaction to anesthetics. ASA classification is III. We performed a general anesthesia with intubation to the patient. Fentanyl $0.25 \mathrm{mg}$, propofol $150 \mathrm{mg}$ and succinylcholine $70 \mathrm{mg}$ were administered for anesthesia induction. After intubation $2 \%$ sevoflurane was used for anesthesia maintenance. The patient was unresponsive to external stimuli for 40 min since the end of the operation. Tree hours after operation, the patient had totally recovered from paralysis and tracheal tube was removed. Four days after operation the patient was discharged from hospital with no special discomfort. Discussion: Reduced plasma cholinesterase activity may occur as a result of inherited, acquired defects or iatrogenic causes. If the acquired defects are excluded low butyrylcholinesterase enzyme (BChE) activity is usually considered to be caused by mutations in butyrylcholinesterase gene (BCHE). There is no specific treatment for butyrylcholinesterase deficiency and the mainstream is to maintain ventilatory support until succinylcholine is metabolized out of the myoneural junction and neuromuscular function recovers. Transfusion of fresh frozen plasma is also viable. Conclusion: There is no specific treatment for plasma cholinesterase deficiency. The best and safest way is to let the patient recover spontaneously. Mechanical ventilation support is very important. Key words: Pseudocholinesterase deficiency, paralysis, ventilatory support
\end{abstract}

\section{Introduction}

Butyrylcholinesterase also known as pseudocholinesterase, serum cholinesterase, plasma cholinesterase, and false cholinesterase was so named due to its ability to hydrolyze butyrylcholine faster than other esters. ${ }^{1}$ Pseudocholinesterase is produced in the liver and found in most tissues, with the exception of red blood cells. The presence of pseudocholinesterase in the body has been established for well over half a century. Although potential functions of this enzyme are debated to this day, its role in the metabolism of choline esters is universally recognized.

Pseudocholinesterase deficiency is a genetic or acquired alteration in the metabolism of choline esters such as succinylcholine, mivacurium, and ester-linked local anesthetics. The most described consequence of pseudocholinesterase deficiency in the literature is prolonged paralysis and apnea after administration of succinylcholine or mivacurium. The latter drug is no longer produced in the United States but is used elsewhere in the world. Less frequently described are adverse outcomes with the use of ester local anesthetics, particularly chloroprocaine. Individuals can live their entire lives with pseudocholinesterase deficiency and not experi- 
ence any untoward health effects, and the presence of the genetic defect is not realized until one is exposed to succinylcholine or mivacurium. ${ }^{2}$

Pseudocholinesterase deficiency, whether inherited or acquired, is an important concept to understand for providers that administer succinylcholine, including anesthesia, intensive care unit (ICU), emergency department, and perioperative personnel. We must appreciate basic concepts of genetics, genetic testing, and pharmacogenetics to make informed choices about our plan of care. We must also be able to extract what diseases, conditions, or medications would cause alterations in a patient's enzyme activity and make thoughtful choices about our care based on this information. Genetically inherited pseudocholinesterase deficiency is typically regarded as an autosomal recessive trait, although it does not adhere to the traditional definition completely. Pseudocholinesterase deficiency is associated with the butyrylcholinesterase or $B C h E$ gene and is located on the long arm of chromosome 3 at $3 q 26.1-26.2$. It is a relatively short piece of genetic material, making DNA testing for the deficiency fairly feasible. ${ }^{3}$

\section{Case report}

Cholinesterase is produced by liver and released into plasma. It is involved in the hydrolysis of ester bonds in muscle relaxants like succinylcholine and several other drugs. It is reported in the case prolonged recovery from paralysis caused by cholinesterase deficiency in a woman who was not diagnosed so preoperatively.

A 70-year-old female patient (weight $48 \mathrm{~kg}$, height $157 \mathrm{~cm}$ ), ASA III, was admitted to our hospital for microlaryngeal surgery. The preoperative evaluation revealed no significant personal or family history for adverse reaction to anesthetics. A general anesthesia was scheduled for this patient. Fentanyl $0.25 \mathrm{mg}$, propofol $150 \mathrm{mg}$ and succinylcholine 70 mg were administered for anesthesia induction. After intubation 2\% sevoflurane was used for anesthesia maintenance. The operation finished successfully within $15 \mathrm{~min}$. The vital signs had been stable during the operation.

The patient was mostly unresponsive to external stimuli for 40 min since the end of the operation. As our hospital did not have a peripheral nerve stimulator, it was hard to accurately assess the extent of neuromuscular blockade. We had to keep stimulating the patient and observed the reaction. Hemodynamic became hypertensive because patient starts waking up, but was not able to move. During the time of recovery we used intermittent sedation with propofol $50 \mathrm{mg}$ as needed to stabilized patients hemodynamic. We used 2 units of fresh frozen plasma. One hour and 30 minutes after operation, the patient coughed when endotracheal tube suction. Two hours after operation the tidal volume of the patient still could not reach 5 $\mathrm{ml} / \mathrm{kg}$. Three hours after operation, the patient had recovered from paralysis and tracheal tube was removed. Four days after operation, the patient was discharged home without special discomfort.

\section{Discussion}

When prolonged neuromuscular blockade happens, a systematic review was necessary to perform. First of all, which kind of neuromuscular relaxant was used? Non-depolarizing or depolarizing, the total dosage, the time of use. In this case, we had used succinylcholine as muscle relaxant during induction. Succinylcholine is commonly used in the surgical rapid-sequence intubation because of its quick effect and short duration of action. ${ }^{4}$ The onset of muscle relaxant after intravenous injection occurs in about $1 \mathrm{~min}$. After a single intubating dose, a complete recovery normally occurs in about $10-12 \mathrm{~min}$. It is hydrolyzed by plasma cholinesterase. ${ }^{5}$ Reduced plasma cholinesterase activity may occur as a result of inherited, acquired defects or iatrogenic causes. ${ }^{6}$ Low BChE activity is associated with hepatic and renal diseases. ${ }^{7,8,9,10,11,12,13,14}$ If the acquired defects are excluded, low BChE activity is usually considered to be caused by mutations in butyrylcholinesterase gene (BCHE). ${ }^{15} \mathrm{BChE}$ deficiency is an autosomal recessive trait. The most important variants are the atypical (A-variants), the Kalow (K-variants), the fluoride (F-variants) and the silent variants. In the White population, the Aand $\mathrm{K}$-variants are the most common. ${ }^{16,17,18,19,20}$

There is no specific treatment for butyrylcholinesterase deficiency and the mainstream is to maintain ventilatory support until succinylcholine is metabolized out of the myoneural junction and neuromuscular function recovers. Transfusion of fresh frozen plasma is also viable. Once a patient was suspected with plasma cholinesterase deficiency, the following measures could be very important: first of all, mechanical ventilation with sedation is necessary 
until succinylcholine is completely metabolized; secondly, succinylcholine must be avoided; thirdly, test of plasma cholinesterase level of the patient and his/her family members is need. We did not manage to measure the plasma cholinesterase level of the patient because she refused to do the test.

\section{Conclusion}

There is no specific treatment for plasma cholinesterase deficiency. The best and safest way is to let the patient recover spontaneously. Mechanical ventilation support is very important. Transfusion of fresh frozen plasma is also viable.

\title{
References
}

1. Manoharan I, Wieseler S, Layer PG, Lockridge O, Boopathy R. Naturally occurring mutation Leu307Pro of human butyrylcholinesterase in the Vysya community of India. Pharmacogenet Genomics. 2006; 16(7):461-468.

2. Manoharan I, Boopathy R, Darvesh S, Lockridge O. A medical health report on individuals with silent butyrylcholinesterase in the Vysya community of India. Clin Chim Acta. 2007; 378(1-2):128-135.

3. Mikami LR, Wieseler S, Souza RL, et al. Five new naturally occurring mutations of the BCHE gene and frequencies of 12 butyrylcholinesterase alleles in a Brazilian population. Pharmacogenet Genomics. 2008; 18(3):213-218.

4. Leadingbam CL (2007) A case of pseudocholinesterase deficiency in the PACU. J Perianesth Nurs 22(4):265-274.

5. Dell DD (1996) Plasma cholinesterase deficiency. J Perianesth Nurs 11(5):304-308.

6. Soliday FK (2010) Pseudocholinesterase deficiency: a comprehensive review of genetic, acquired, and drug influences. J Am Assoc Nurse Anesth 78(4):313-320.

7. Aldrete JA, O'Higgins JW, Holmes J. Changes of plasma cholinesterase activity during orthotopic liver transplantation in man. Transplantation. 1977; 23(5):404-406.

8. Mullan DP, Williams NE. Left-sided amoebic liver abscess with pulmonary involvement, pericarditis, and pseu do cho lin es ter ase deficiency. Br Med J. 1965; 1(5429):235-236.

9. McQueen MJ. Clinical and analytical considerations in the utilization of cholinesterase measurements. Clin Chim Acta. 1995; 237(1-2):91105.

10. Whittaker M. Plasma cholinesterase variants and the anaesthetist. Anaesthesia. 1980; 35(2):174-197.

11. Simon NM, Del Greco F, Dietz AA, Rubinstein HM. Serum cholinesterase deficiency in renal failure. Trans Am Soc Artif Intern Organs. 1969;15(1):328-332.

12. Bishop MJ, Hornbein TF. Prolonged effect of succinylcholine after neostigmine and pyridostigmine administration in patients with renal failure. Anesthesiology. 1983; 58(4):384-386.

13. Ryan DW. Preoperative serum cholinesterase concentration in chronic renal failure: clinical experience of suxamethonium in 81 patients undergoing renal transplant. Br J Anaesth. 1977; 49(9):945-949.

14. Ryan DW. Postoperative serum cholinesterase activity following successful renal transplantation. Br J Anaesth. 1979; 51(9):881-884.

15. Wichmann S, Fжrk G, Bundgaard JR, Gдtke MR (2016) Patients with prolonged effect of succinylcholine or mivacurium had novel mutations in the butyrylcholineesterase gene. Pharmacogenet Genomics 26(7):351-356.

16. Kalow W, Staron N. On distribution and inheritance of atypical forms of human serum cholinesterase, as indicated by dibucaine numbers. Can J Biochem Physiol. 1957; 35(12):1305-1320.

17. Kalow W, Genest K. A method for the detection of atypical forms of human serum cholinesterase: determination of dibucaine numbers. Surv Anesthesiol. 19; 17(6):581-588.

18. Morgan GE, Mikhail MS, Murray MJ. Clinical Anesthesiology. 4th ed. New York, NY: McGraw-Hill; 2006:205-226.

19. Harris H, Whittaker, M. Differential inhibition of human serum cholinesterase with fluoride: recognition of two new phenotypes. Nature. 1961; 191:496-498

20. Bartels CF, Jensen FS, Lockridge O, et al. DNA mutation associated with the human butyrylcholinesterase K-variant and its linkage to the atypical variant mutation and other polymorphic sites. Am J Hum Genet. 1992; 50(5):1086-1103.

\author{
Correspondence to: \\ Tsvetomir Marinov, $\mathrm{MD}, \mathrm{PhD}$ \\ Department of Anesthesiology and Intensive Care \\ University Hospital „Queen Giovanna” - ISUL \\ Byalo More Str 8 \\ Medical University - Sofia \\ E-mail:ts.marinov@abv.bg
}

